\title{
IFCCDC: A Fuzzy Control based Congestion Detection and Control in Wireless Sensor Networks
}

\author{
Rekha Chakravarthi \\ Research Scholar, Sathyabama University
}

\author{
C.Gomathy \\ PhD ,Prof \& Head ,Dept of Electronics \& Control \\ Engineering , \\ Sathyabama University
}

\begin{abstract}
In Wireless Sensor Networks (WSN's), congestion plays an important role in degrading the performance of the network Under idle condition, the network load is very low whereas when an event is detected the network load becomes high which leads to congestion. Due to congestion the overall performance of the network degrades. Hence it is necessary to detect and control congestion. In this paper an efficient technique to detect and control congestion has been proposed. The congestion is detected by calculating a metric called Congestion Degree (Cd). It is the ratio between packet inter arrival time and packet inter service time. Once the congestion is detected, it is notified using Implicit Congestion Notification (ICN) signaling. On receiving the congestion notification signal, the transmission rate is controlled in order to reduce congestion. Further congestion control is implemented using Fuzzy Logic Controller. The performance of the network is measured for delivery ratio with different transmission rate and the PDR is compared with CODA.
\end{abstract}

\section{General Terms}

Wireless Sensor Network, Fuzzy Logic , Algorithm , Congestion Control.

\section{Keywords}

Wireless Sensor Networks (WSNs), Congestion, Congestion Degree $\left(\mathrm{C}_{\mathrm{d}}\right)$, Fuzzy Logic Controller .

\section{INTRODUCTION}

The Wireless Sensor Networks (WSNs) consists of several sensor nodes which will monitor the environmental conditions such as temperature, pressure, vibration, etc. Basically the sensor nodes sense the information and process the information. Then they transmit the information as data packets to the sink node. When an event occurs in the network, large number of sensor nodes will report their data packets to sink. When large number of sensor nodes are active in transmitting the data packets the load becomes heavy and traffic will also increases. This might leads to congestion in the network.

The very important issue in the WSNs is congestion. There are various reasons for congestion. They are Buffer overflow, Channel contention, Packet collision, Many-to-one nature, etc. [11]. Buffer overflow occurs when the number of incoming packets is greater than the available buffer space. [12] Channel contention occurs between different flows and different packets of a flow. Packet collisions indicate lower level congestion and leads to packet drops. The many-to-one nature of event communication between multiple sources and sink causes bottleneck around sink.

\section{RELATED WORK}

In [1], to detect congestion each node calculates its node rank based on the parameters buffer Size, hop count, channel busy ratio and MAC overhead. When the node rank crosses a threshold value $\mathrm{T}$, the sensor node will set a congestion bit in every packet it forwards. If the congestion bit is set, the downstream node calculates the Rate Adjustment Feedback based on the rank and propagates this value upstream towards the source nodes. The source nodes will adjust their transmission rates dynamically based on this feedback. In Hop-by-Hop congestion control technique [2], the Effective Queue Length (EQL) is measured. EQL is used to determine whether the congestion is occurred or not. The technique called CONSEQ (CONtrol of Sensor Queues) is used to control the congestion. The CONSEQ aims to reduce congestion and, thereby, decrease the delay and energy consumption due to packet losses and retransmission in WSN.

In [3], CODA, an energy efficient congestion control scheme for sensor networks was proposed. CODA (COngestion Detection and Avoidance) comprises three mechanisms: (i) receiver-based congestion detection; (ii) open-loop hop-byhop backpressure; and (iii) closed-loop multi-source regulation. CODA detects congestion based on queue length as well as wireless channel load at intermediate nodes. Furthermore it uses explicit congestion notification approach and also an AIMD rate adjustment technique. In [4], the author proposes a Prioritized Heterogeneous Traffic-oriented Congestion Control Protocol (PHTCCP) to control congestion. It uses packet service ratio to detect congestion. Packet service ratio is defined as the ratio of average packet service rate and packet scheduling rate in each sensor node. PHTCCP uses hop-by-hop rate adjustment for controlling the congestion. The output rate of a node is controlled by adjusting the scheduling rate. 
In [5], the congestion is detected by measuring the length of the queue. The queue length increases when the Packet interarrival time is more than Packet inter-service time. Using queue length, the Buffer Occupancy is calculated. When the Buffer Occupancy increases, the congestion increases. Congestion is controlled by assigning priority to the data packets. When the Buffer Occupancy increases, the data packets are dropped depending on priority assigned to the data packets i.e., Intelligent Packet Dropping. In [6], the author proposes a Minimum Hop Disjoint Multipath routing algorithm with Time Slice load balancing congestion control scheme (MHDMwTS) to ensure reliability in Wireless Multimedia Sensor Networks (WMSNs). In this, congestion detection is done by monitoring the reception queue of nodes. The congestion is controlled by adjusting the transmission rate at source nodes.

In [7], congestion is detected by measuring the queue length. The congestion is controlled by using three techniques i) hopby-hop flow control, ii) source rate limiting, and iii) prioritized MAC. Even in high offered load it claims to achieve good throughput and fairness. In [8], the authors propose a hop-by-hop congestion control technique, Congestion Control and Fairness (CCF), which uses packet service time to infer the available service rate and therefore detects congestion in each intermediate sensor node. CCF ensures simple fairness. However, it lacks efficient utilization of the available link capacity when some nodes do not have any traffic to send or nodes remaining in sleep mode or the nodes whose flows do not pass through the congested area.

PCCP [9] is a recent congestion control protocol for WSNs which uses hop-by-hop approach for rate control. PCCP is a node priority based congestion control protocol which allows sensor nodes to receive priority-dependent throughput. However, PCCP does not have any mechanism for handling prioritized heterogeneous traffic originated from a single node. In [10], congestion is detected by calculating depth of congestion at the sink node. To calculate depth of congestion service and arrival rate are used. It's the ratio of local packet inter-service rate and local packet inter-arrival rate. Hop-byHop Rate control Technique (HRCT) is implemented to control congestion. The sensor/transmission rate of the node is adjusted based on depth of congestion and overall sensor priority.

\section{PROPOSED WORK}

In this section, Fuzzy Logic Controller has been implemented to control congestion due to concurrent transmission. Congestion is detected by calculating Congestion Degree $\left(\mathbf{C}_{\mathrm{d}}\right)$. If the measured congestion degree is greater than predefined threshold, it intimates that the congestion has been occurred; else there is no congestion in the network. Once the congestion is detected, the congestion notification signal is sent to intermediate nodes. For this purpose the Implicit Congestion Notification (ICN) signalling is used. Upon receiving the congestion notification signal each node adjusts its transmission rate. Fig. 1 shows the architecture of the proposed technique.

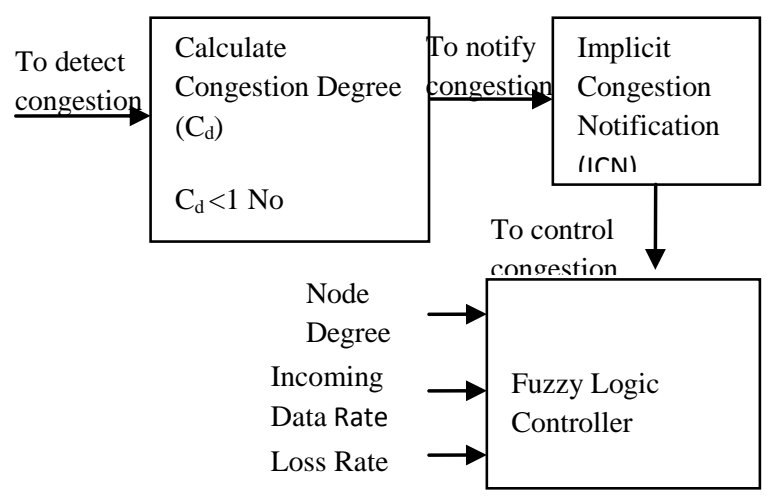

Fig.1: Architecture of proposed technique

\subsection{To Calculate Congestion Degree $\left(\mathbf{C}_{\mathrm{d}}\right)$}

Congestion Degree is the ratio between Local packet interarrival time and Local packet inter-service time. The measured value of congestion Degree indicates the level of congestion of the network.

Congestion Degree $\left(\mathrm{C}_{\mathrm{d}}\right)=\mathrm{T}_{\mathrm{s}} / \mathrm{T}_{\mathrm{a}}$

Where $\mathrm{T}_{\mathrm{s}}=$ local packet inter-service time

$\mathrm{T}_{\mathrm{a}}=$ local packet inter-arrival time

One the Congestion Degree $\left(\mathbf{C}_{\mathbf{d})}\right.$ is calculated, the measured value is compared with threshold value $\left(T_{h}\right)$. Here the threshold is set as one. When congestion degree value is greater than or equal to threshold value, then it infers congestion has occurred. When congestion degree value is less than threshold value, then it infers there is no congestion.

$\mathbf{C}_{\mathbf{d}}<\mathrm{T}_{\mathrm{h}} ;$ No Congestion

Packet arrival is slower than departure

$\mathbf{C}_{\mathbf{d}} \geq \mathrm{T}_{\mathrm{h}} ;$ Congestion

Packet arrival is faster than departure.

When packet arrival is slower than departure then there is no backlogged packets, thus there is no congestion. But when packet arrival is faster than departure then it increases the backlogged packets which lead to congestion .

\subsection{Implicit Congestion Notification}

Once the congestion is detected, the notification signal is sent to intermediate nodes. There are two types of congestion notification signal. They are Implicit Congestion Notification (ICN) and Explicit Congestion Notification (ECN). In ICN, the notification signal is sent along with the data packet. In ECN, the notification signal is sent as separate packet. In the proposed work Implicit Congestion Notification is used to send notification signal to all nodes in the network. The signal is send along with the data packets to nearby nodes. On receiving the signal, each node adjusts its transmission rate. ICN is an effective technique than ECN, because in ECN the notification is sent as separate packet. So this makes heavy traffic in the network which leads to congestion. 


\subsection{Fuzzy Logic Controller}

In WSNs, fuzzy logic is used for improving decision-making, reduce resource consumption, and increase performance of the network. Some of the areas it has been applied to are clusterhead election [11], [13], security [12], [14], data aggregation [15], routing [16], [17], MAC protocols [18], and QoS [19], [20]. However, many existing system have not implemented fuzzy logic to control congestion. This paper deals with Fuzzy Logic Controller to control congestion.

. A Fuzzy system basically consists of three parts: fuzzifier, inference scheme, and defuzzifier. [21] The fuzzifier maps each crisp input value to the corresponding fuzzy sets and thus assigns it a truth value or degree of membership for each fuzzy set. The fuzzified values are processed by the inference scheme, which consists of a rule base and various methods for inferring the rules. The rule base is simply a series of IFTHEN rules that relate the input fuzzy variables with the output fuzzy variables using linguistic variables, each of which is described by a fuzzy set, and fuzzy implication operators AND, OR etc. All the rules in the rule-base are processed in a parallel manner by the fuzzy inference scheme. Any rule that fires contributes to the final fuzzy solution space. The inference rules govern the manner in which the consequent fuzzy sets are copied to the final fuzzy solution space. The defuzzifier performs defuzzification on the fuzzy solution space. That is, it finds a single crisp output value from the solution fuzzy space.

The main objective of our fuzzy routine is to adjust the transmission rate of the sensor nodes, so that the performance of the network is improved. The performance of the network can be improved by reducing the packet drop and increasing the network throughput. Thus, the fuzzy rule base has been turned so as to not only decrease packet drop but also to increase the network throughput. The fuzzy rule base has been shown in the Table 1.

Table 1. Fuzzy Rule Base

\begin{tabular}{|c|c|c|c|c|}
\hline Rule & $\begin{array}{l}\text { Incoming } \\
\text { Data Rate }\end{array}$ & $\begin{array}{l}\text { Node } \\
\text { Degree }\end{array}$ & $\begin{array}{l}\text { Loss } \\
\text { Rate }\end{array}$ & Rate \\
\hline 1. & L & L & L & IH \\
\hline 2 & L & L & M & IM \\
\hline 3. & L & L & H & DM \\
\hline$\cdot$ & $\cdot$ & $\cdot$ & $\cdot$ & \\
$\cdot$ & $\cdot$ & $\cdot$ & $\cdot$ & $\cdot$ \\
\hline 27. & H & H & H & DH \\
\hline
\end{tabular}

The fuzzy rules are framed by using three input linguistic variables namely Incoming Data Rate, Node Degree and Loss
Rate. The values for input linguistic variables are Low (L), Medium (M) and High (H). Therefore the rule base consists of 27 rules. In this, there is a single output fuzzy variable, namely Rate, which is the defuzzified value that adjusts the transmission rate of the sensor nodes. The value for fuzzy output variable is Increased High (IH), Increased Minimum (IM), Decreased High (DH) and Decreased Minimum (DM).

The congestion is controlled by using the Fuzzy Rule Base. Once the node receives the congestion notification signal it adjusts its transmission rate based on fuzzy rules.

\section{Algorithm Description}

- Setup initial sensor nodes in the network.

- Assign the capability to all nodes to sense the data.

- Set the transmission rate for each node.

- Assign the Congestion Degree $\left(C_{d}\right)$ threshold value as 1 and current value as 0 .

while

Data packets are transmitted and received by the node.

do

Calculate Congestion Degree $\left(C_{d}\right)$.

$$
C_{d}=T_{s} / T_{a}
$$

if

Congestion Degree $\left(C_{d}\right)$ is less than threshold

then

Packet arrival rate is less than departure rate.

No Congestion in the network.

else

Packet arrival rate is greater than departure rate.

Congestion has occurred.

Transmission rate is adjusted based on fuzzy rules.

Congestion is controlled.

end if.

end while 


\section{SIMULATION STUDY}

\subsection{Simulation Settings}

A Wireless Sensor Networks (WSNs) which consists of 200 sensor nodes in $1000 \mathrm{~m} \times 1000 \mathrm{~m}$ area is created. The number of active sources varies from 4 to 8 . The data packets are transmitted and received by the nodes using Ad-hoc On Demand Distance Vector (AODV) routing protocol. When an event is detected the sensor nodes transmits more number of data packets. When more number of nodes try to seize the same channel the congestion will occur. This is known as channel contention. Once the congestion is detected the each intermediate sensor node adjusts its transmission rate in order to control the congestion. To detect and control congestion an effective and efficient technique is implemented in NS2 Simulator. The network performance is evaluated by calculating delay and packet delivery ratio. A summary of simulation parameters is given in Table 2 .

Table 2. Simulation Parameters

\begin{tabular}{|l|l|}
\hline Channel Type & Channel/Wireless Channel \\
\hline Radio-Propagation & Propagation/Two RayGround \\
\hline Network interface & Phy/WirelessPhy \\
\hline MAC Protocol & Mac/802_11 \\
\hline Interface Queue & Queue/Drop Tail/PriQueue \\
\hline Antenna Model & Antenna/Omni Antenna \\
\hline Routing Protocol & $\begin{array}{l}\text { AODV(Ad-hoc On Demand } \\
\text { Distance Vector) }\end{array}$ \\
\hline Simulation start time & 1.0 \\
\hline Simulation Stop time & 50 \\
\hline Packet size & 512 \\
\hline Number of nodes & 200 \\
\hline Total area & $1000 \mathrm{x} 1000$ \\
\hline Active source & $4-8$ \\
\hline Buffer size & $10 \mathrm{~kb}-30 \mathrm{~kb}$ \\
\hline Number of sink & $150 \mathrm{packets}$ \\
\hline
\end{tabular}

\subsection{Simulation Scenario}

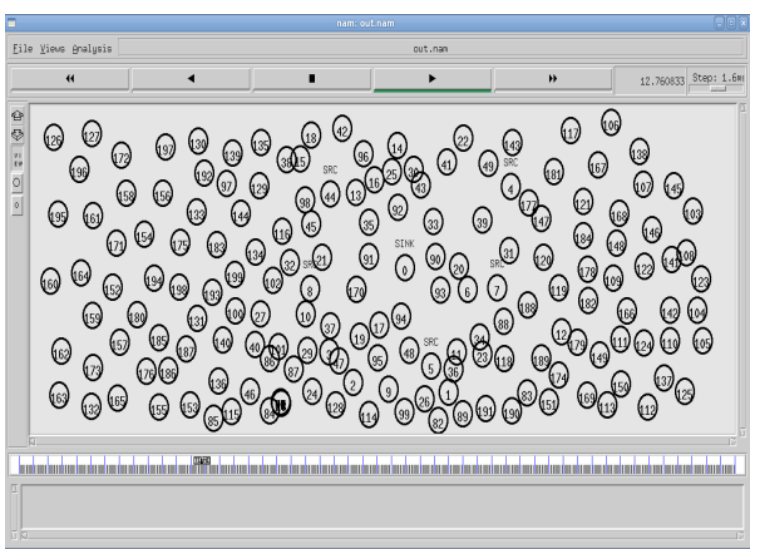

Fig.2: Scenario with data transmission from 5 sources to 1 sink.

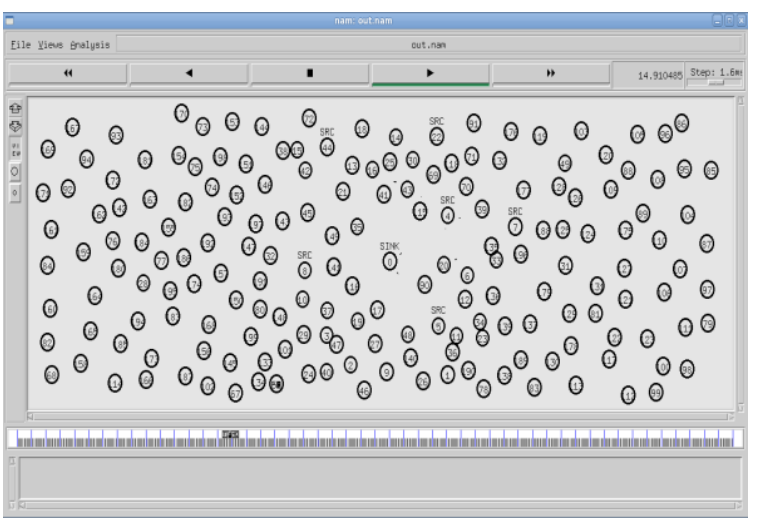

Fig.3: Scenario with data transmission from 6 sources to 1 sink.

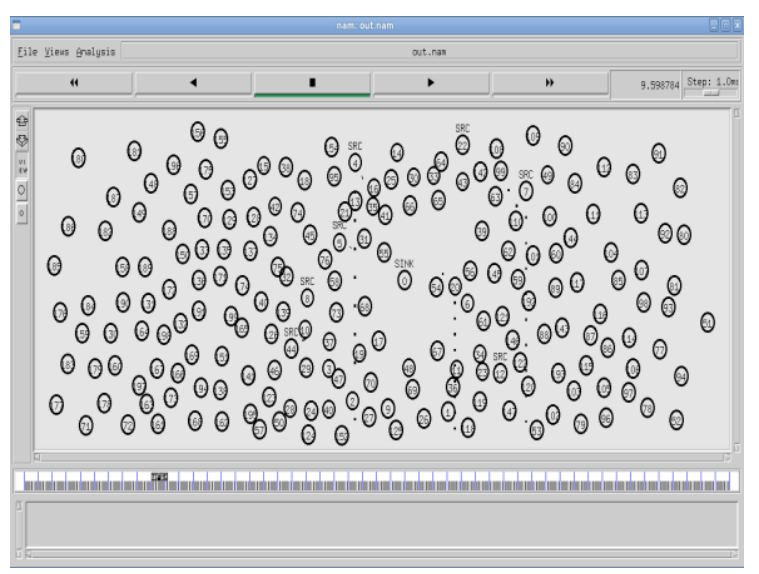

Fig.4: Scenario with data transmission from 7 sources to 1 sink .

Fig. 2 shows the scenario of data packet transmission from 5 sources to 1 sink with a network size of 200 sensor nodes . Fig. 3 shows the scenario of data packet transmission from 6 sources to 1 sink with a network size of 200 sensor nodes and fig. 4 shows the scenario of data packet transmission from 7 sources to 1 sink. 


\subsection{Simulation Results}

In this paper, an analysis is done for PDR and Delay and the result is compared with CODA. The simulation result shows the effectiveness of the proposed technique.

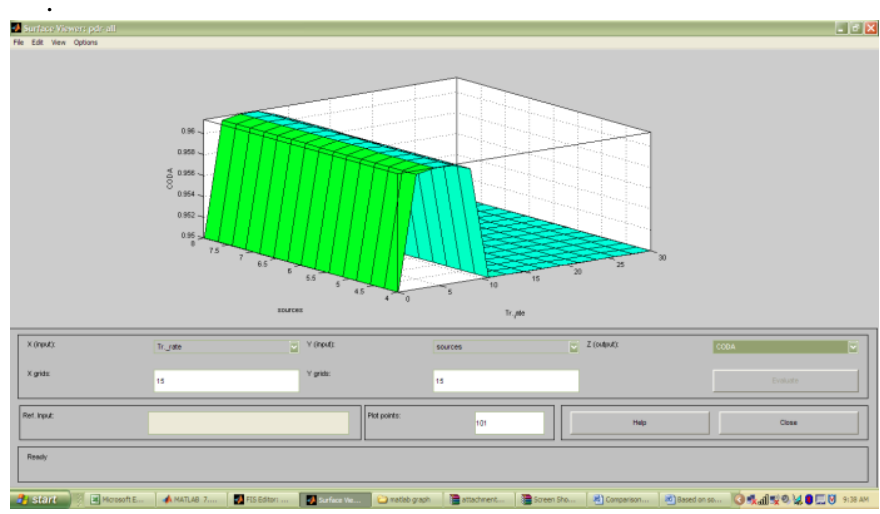

Fig.5: Packet Delivery Ratio for CODA

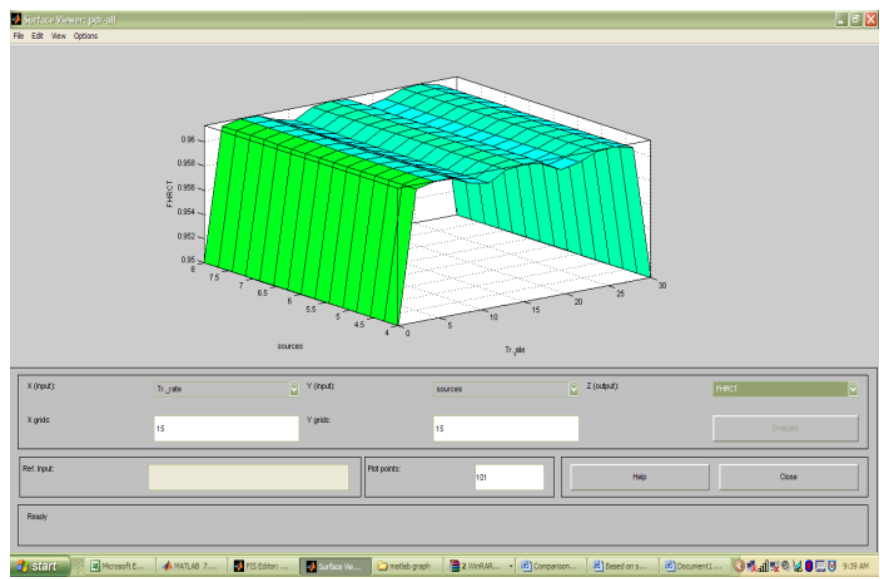

Fig.6: Packet Delivery Ratio for FHRCT

Fig. 5 and Fig. 6 shows the simulation result of Packet Delivery Ratio Vs Transmission rate. The PDR of FHRCT is high when compared to CODA .The packet delivery ratio is $100 \%$ using Fuzzy HRCT for lower transmission rate and it reduces to $98.5 \%$ for higher transmission rate whereas packet delivery ratio is $93 \%$ using CODA for less transmission rate and it reduces to $95.5 \%$ for high transmission rate.

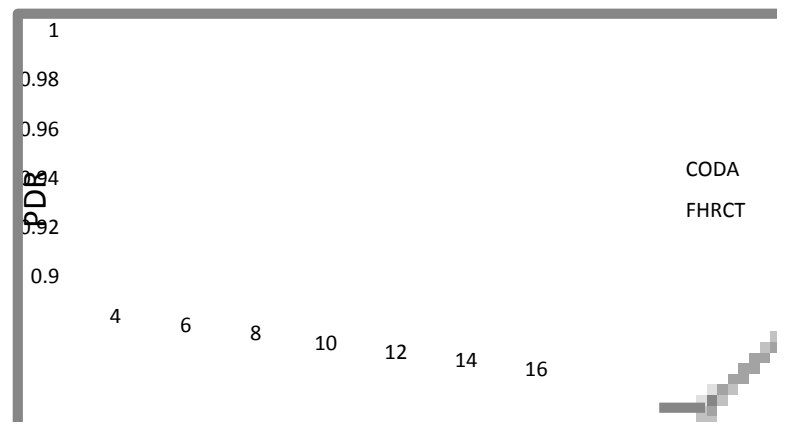

Fig.7: Comparison of PDR for CODA and FHRCT

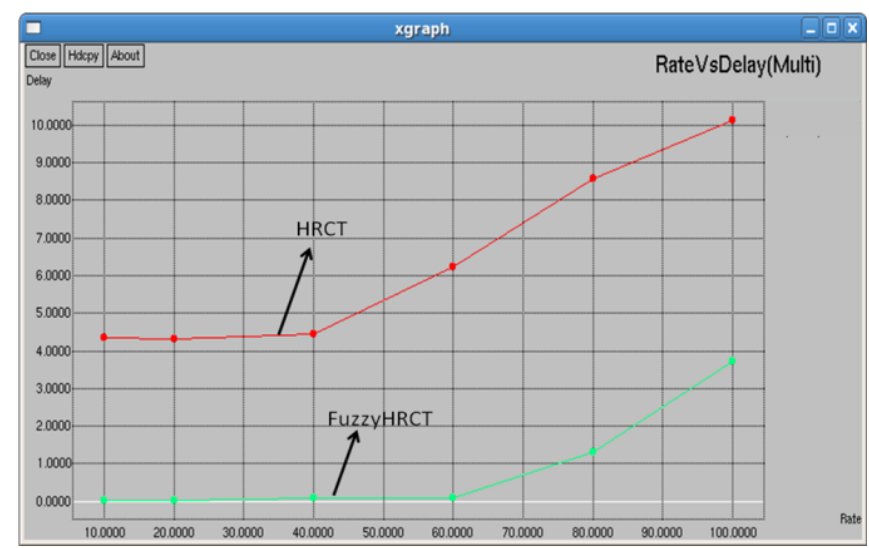

Fig.8: Delay Vs. Transmission rate

Fig. 8 shows the simulation result of Delay Vs. Transmission rate. The Delay of Fuzzy HRCT is zero for less transmission rate and $3.8 \mathrm{sec}$ for higher transmission rate whereas delay is $4.5 \mathrm{sec}$ using HRCT for lower transmission rate and $11 \mathrm{sec}$ for higher transmission rate

Thus a comparison is done for Packet Delivery Ratio between for CODA and FHRCT. This paper concludes that the technique implemented in FHRCT is more efficient when compared to previous technique in controlling congestion. A comparison is also made for delay between HRCT and FHRCT which concludes that the time delay taken for FHRCT is very less.

\section{REFERENCES}

[1] Kamal Kumar Sharma, Dr. Harbhajan Singh and Dr. R.B Patel "A Hop by Hop Congestion Control Protocol to Mitigate Traffic Contention in Wireless Sensor Networks" , in Proceedings of International Journal of Computer Theory and Engineering, Vol.2, No.6, pp 1793-8201, December, 2010.

[2] Basaran, kyoung-Don Kang, Mehmet H. Suzer "Hop-byHop Congestion Control and Load Balancing in Wireless Sensor Networks", in proceedings of 2010 IEEE $35^{\text {th }}$ conference on Local Computer Networks, pp. 448-455, 2010.

[3] Chieh-Yih Wan, Shane B. Eisenman,Andrew T. Campbell," Energy-Efficient Congestion Detection and Avoidance in Sensor Networks ",in proceedings of ACM Transactions on Sensor Networks, Vol. 7, No. 4, Article 32,pp.32.1-32.312011.

[4] Muhammad Monowar, Obaidur Rahman, Al-Sakib Khan Pathan, and Choong Seon Hong "Prioritized Heterogeneous Traffic-Oriented Congestion Control Protocol for WSNs" in proceedings of The International Arab Journal of Information Technology, Vol. 9, No. 1 pp. 39-48, 2012.

[5] Rekha Chakravarthi, C.Gomathy "IPD: Intelligent Packet Dropping Algorithm for Congestion Control in Wireless Sensor Network", in Proceedings of IEEE, pp. 222-225, 2010.

[6] Guannan Sun, Jiandong Qi, Zhe Zang, Qiuhong Xu "A Reliable Multipath Routing algorithm with related congestion control scheme in Wireless Multimedia 
Sensor Networks", in Proceedings of IEEE, pp. 229233, 2011.

[7] Hull B., Jamieson K., and Balakrishnan H., "Mitigating Congestion in Wireless Sensor Networks," in Proceedings of the $2^{\text {nd }}$ International Conference on Embedded Networked Association for Computing Machinery Sensor Systems, USA, pp. 134-147, 2004.

[8] Ee C. and Bajcsy R., "Congestion Control and Fairness for Many-to-One Routing in Sensor Networks," in Proceedings of the $2^{\text {nd }}$ International Conference on Embedded Networked Association for Computing Machinery Sensor Systems, USA, pp. 148-161, 2004.

[9] R.Then Malar "Congestion Control in Wireless Sensor Networks Based Multi-Path Routing In Priority Rate Adjustment Technique “, in proceedings of International Journal of Advanced Engineering \& Applications, pp 2833, 2010.

[10] Rekha Chakravarthi, C. Gomathy “ Hop-by-Hop Rate Control Technique for Congestion Due to Concurrent Transmission in Wireless Sensor Network" in proceedings of World of Computer Science and Information Technology Journal (WCSIT) ISSN: 22210741 Vol. 1, No. 8,pp. 351-356, 2011.

[11] J. Kim, S. Park, Y. Han, and T. Chung, "CHEF: Cluster Head Election Mechanism Using Fuzzy Logic In Wireless Sensor Networks", in proceedings of ICACT, pp. 654-659, 2008.

[12] H. Lee and T. Cho, "Fuzzy Logic Based Key Disseminating In Ubiquitous Sensor Networks," in proceedings of ICACT, pp. 958-962, 2008.

[13] I. Gupta, D. Riordan, and S. Sampalli, "Cluster-Head Election Using Fuzzy Logic for Wireless Sensor Networks," in proceedings of CNSR, pp. 255-260, 2005.

[14] B. Kim, H. Lee, and T. Cho, "Fuzzy Key Dissemination Limiting Method for the Dynamic _Ltering-Based Sensor Networks," in proceedings of ICIC, pp. 263-272, 2007.

[15] B. Lazzerini, F. Marcelloni, M. Vecchio, S. Croce, and E. Monaldi, "A Fuzzy Approach To Data Aggregation To Reduce Power Consumption In Wireless Sensor Networks," in proceedings of NAFIPS, pp. 436-441, 2006.

[16] J. Kim and T. Cho, "Routing Path Generation for Reliable Transmission in Sensor Networks Using GA with Fuzzy Logic Based_Tness Function," in proceedings of ICCSA, pp. 637-648, 2007.
[17] S.-Y. Chiang and J.-L. Wang, "Routing Analysis Using Fuzzy Logic Systems in Wireless Sensor Networks," in proceedings of KES, pp. 966-973, 2008.

[18] F. Xia, W. Zhao, Y. Sun, and Y.-C. Tian, "Fuzzy Logic Control Based QoS Management in Wireless Sensor/Actuator Networks," pp. 3179-3191, 2007.

[19] S. A. Munir, Y. W. Bin, R. Biao, and M. Jian, "Fuzzy Logic Based Congestion Estimation For Qos In Wireless Sensor Network," in proceedings of WCNC, pp. 43364341,2007

[20] F. Xia, W. Zhao, Y. Sun, and Y.-C. Tian, "Fuzzy Logic Control Based QoS Management in Wireless Sensor/Actuator Networks," pp. 3179-3191, 2007.

[21] Tarique Haider, Mariam Yusuf "A Fuzzy Approach to Energy Optimized Routing for Wireless Sensor Networks", in Proceedings of The International Arab Journal of Information Technology, Vol. 6, No. 2, pp. 179-188, 2009.

\section{AUTHORS PROFILE}

Rekha Chakravarthi acquired a B.E. degree in Electronics and Communication Engineering from Sathyabama Engineering College, Chennai and an M.E. degree in Applied Electronics Engineering from Sathyabama University, in 2004. She is pursuing Ph.d in Sathyabama University, Chennai. She has published over 9 research papers in National and International conferences and Journals. Her areas of interest include wireless sensor networks, wireless/mobile networks and high performance networks.

C. Gomathy acquired a B.E. (with honors) degree in Electronics and Communication Engineering from Government College of Engineering, Tirunelveli, in the year 1986, and an M.S. degree in Electronics and Control Engineering from Birla Institute of Technology and Science, Pilani, in 1992. She also obtained an M.S. (by research) degree from Anna University in 2001. She obtained her Ph.D. in the area of Mobile Ad hoc networks from Department of Electronics and Communication Engineering, College of Engineering, Anna University, Chennai, India, in the year 2007. She has published over 34 research papers in National and International conferences and journals. Her areas of interest include mobile ad hoc networks, high-speed networks, and wireless sensor networks. 